\title{
Tsafon
}

Revue d'études juives du Nord

$80 \mid 2020$

Varia

\section{Le bouc émissaire selon Pierre Lasry}

\section{Simone Grossman}

\section{OpenEdition}

Journals

Édition électronique

URL : https://journals.openedition.org/tsafon/3252

DOI : $10.4000 /$ tsafon.3252

ISSN : 2609-6420

\section{Éditeur}

Association Jean-Marie Delmaire

\section{Édition imprimée}

Date de publication : 1 décembre 2020

Pagination : 83-96

ISSN : 1149-6630

\section{Référence électronique}

Simone Grossman, «Le bouc émissaire selon Pierre Lasry », Tsafon [En ligne], 80 | 2020, mis en ligne

le 01 décembre 2020, consulté le 24 juin 2021. URL : http://journals.openedition.org/tsafon/3252 ;

DOI : https://doi.org/10.4000/tsafon.3252 


\title{
Le bouc émissaire selon Pierre Lasry
}

\author{
Simone Grossman * \\ Est-ce que ce bouquin émissaire \\ touche à l'autel du sacrifice ?
}

(P. Lasry)

En 2010, le cinquantième anniversaire de la communauté sépharade de Montréal a été marqué par la parution de l'Anthologie des écrivains sépharades du Québec ${ }^{1}$. Les Sépharades, originaires principalement du Maroc, arrivés à Montréal dans les années soixante, ont formé la troisième vague d'immigration juive au cours du vingtième siècle $^{2}$. En tant que francophones, ils ont contribué au rapprochement entre les Québécois de souche et la communauté juive anglophone. De nos jours, ils participent activement à la vie culturelle du Québec ${ }^{3}$. La présente étude porte sur trois romans de Pierre Lasry figurant en bonne place dans l'Anthologie: Une Juive en Nouvelle-France (2004) ${ }^{4}$, Don Juan et les moulins à vents $(2008)^{5}$ et L'homme qui n'avait rien à dire $(2011)^{6}$. Arrivé à Montréal en 1957, Lasry a été cinéaste à l'Office

\footnotetext{
* Université Bar Ilan, Ramat-Gan, Tel-Aviv, Israël.

${ }^{1}$ David Bensoussan (dir.), Anthologie des écrivains sépharades du Québec, Montréal, Éditions du Marais, 2010. Les 656 pages de l'Anthologie incluent des essais et des extraits d'œuvres littéraires.

2 Voir l'ouvrage de Pierre Anctil, Trajectoires juives au Québec, Québec, Presses Universitaires de Laval, 2010.

${ }^{3}$ Voir Yolande Cohen (dir.), Les Sépharades du Québec. Parcours d'exils nordafricains, Montréal, Del Busso Éditeur, 2017.

${ }^{4}$ Pierre Lasry, Une Juive en Nouvelle-France, Montréal, Éditions Midbar, 2004 [2000]. Traduit en anglais par l'auteur sous le titre Esther: A Jewish Odyssey, Montréal, Éditions Midbar, 2002. Désigné ultérieurement sous le sigle JNF.

${ }^{5}$ P. Lasry, Don Juan et les moulins à vents, Montréal, Éditions du Marais, 2008. Désigné ultérieurement sous le sigle DJ.

6 P. Lasry, L'homme qui n'avait rien à dire. Portrait de l'auteur en meurtrier pornographe, Montréal, Éditions Midbar, 2011. Désigné ultérieurement sous le sigle HD.
} 
national du film du Canada avant de devenir écrivain. Comme chez d'autres écrivains migrants d'origine marocaine, l'identité, l'Histoire et la mémoire sont les thématiques majeures de ses romans ${ }^{7}$. Dans la préface à l'Anthologie, Anne-Elaine Cliche souligne que Lasry puise «aux plus riches strates de l'histoire du marranisme [...] pour les inscrire dans le terreau québécois dont il manie aussi bien les figures que la langue $»^{8}$. La figure du bouc émissaire occupe une place centrale dans son œuvre. Représenté sous divers aspects dans les films documentaires de Lasry, le Juif bouc émissaire est au centre de son film Shylock ${ }^{9}$. Lasry déconstruit l'image historique du Juif déicide, opérant un retour sur la haine séculaire de l'Église. Passant en revue les diverses interprétations du personnage de Shakespeare dans des contextes géographiques, temporels et politiques différents, il montre que l'usurier juif cruel et sanguinaire du Marchand de Venise est victime des chrétiens qui lui font porter leurs péchés.

En 2007, dans un article intitulé «Le bouc émissaire », Lasry analyse la signification historique, religieuse et sociale du bouc émissaire en s'étayant sur Le Rameau d'or de J.G.Frazer ${ }^{10}$. Rappelant que « dans toutes les sphères humaines il y a une clause parfois invisible, parfois visible pour la sélection ou le rejet de certains individus », il assimile le "désir d'éliminer certains individus de la sphère socio-économique, ethnique et raciale» au comportement animal, arguant que les « comportements humains violents » sont « le reliquat d'un mécanisme de survie localisé dans notre cerveau reptilien humain ». Quand la société se sent en danger, expose-t-il, elle sacrifie ses membres les plus faibles, «des êtres différenciés, étrangers souvent, des Juifs, des Noirs, des femmes » ou des handicapés, dans l'espoir d'« endiguer [...] la menace sociale ou politique ». Le judaïsme, dit-il, vise à « contrôler l'instinct de la violence » pour « le transformer en un comportement de compassion et de raisonnement éthique ». Dans l'Ancien Testament, le bouc émissaire était envoyé au désert pour expier les fautes du peuple juif. Jésus, " auto offert en victime sacrificielle pour absoudre tous les péchés futurs », est

\footnotetext{
${ }^{7}$ Voir Mechtild Gilzmer, «Littérature migrante francophone d'origine marocaine au Québec », Zeitschrift für Kanada-Studien 27.2, 2007, p. 9-29.

${ }^{8}$ Anne-Elaine Cliche, préface à l'Anthologie des écrivains sépharades du Québec, p. 20.

${ }^{9}$ P. Lasry, Shylock, National Film Board of Canada, 1999. Nominé pour le prix Gemini du meilleur film en 2001. (Voir les commentaires dans NY Times, Wall Street Journal, Washington Post, Miami Herald le 24 avril 2000).

${ }^{10}$ P. Lasry, «Le bouc émissaire» / « Scapegoat», JMag-Magazine culturel de la communauté juive de Montréal-La Voix Sépharade, novembre 2005. Les citations suivantes sont extraites du même article.
} 
pour Lasry une régression justifiant le sacrifice humain et les «wagons pleins à craquer de boucs émissaires juifs roulant grièvement sur les rails menant aux fours crématoires de la Shoah ». La violence humaine, qu'elle se manifeste sous forme de lynchage, de pogrome ou de sorcières brûlées, se rapporte en droite ligne, selon lui, au syndrome du bouc émissaire. L'article se clôt sur le modèle d'Abraham, «le premier à placer la vie humaine au centre de son système éthique, en apprenant à subjuguer ses propres instincts primitifs ${ }^{11}$.

\section{La perspective intergénérationnelle sur le bouc émissaire dans Une Juive en Nouvelle-France}

L'action d'Une Juive en Nouvelle-France, roman historique basé sur des faits réels, se déroule en France et en Nouvelle-France au XVIII ${ }^{\mathrm{e}}$ siècle, époque où le christianisme dominant imputait la mort de Jésus à toutes les générations des Juifs, passées et futures. L'aventure authentique d'Esther Brandeau est attestée par les historiens. Le roman s'ouvre sur deux prologues successifs, espagnol et français, où sont décrits les supplices de Juives condamnées par l'Inquisition à être brûlées. Le premier prologue, situé et daté à « Barcelone, février 1395 », relate les derniers moments d'une ancêtre d'Esther Brandeau. Avant de mourir, elle assume son destin de bouc émissaire : «Ils ont placé tous leurs péchés en moi pour s'en débarrasser » (JNF 16). Consciente des enjeux théologiques, elle condamne l'Église dans le poème où elle affirme sa foi dans l'avenir du peuple juif :

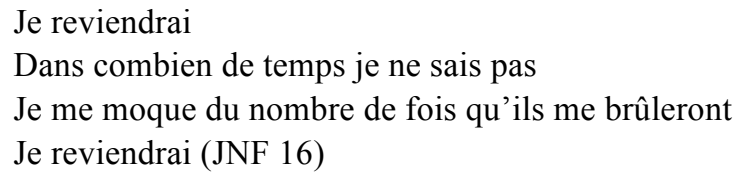

Le second prologue relate le martyre de Catherine de Fernandès, brûlée à Saint-Jean-de-Luz en 1622 pour avoir recraché 1'hostie. Esther Brandao $^{12}$, élevée au couvent jusqu'à son adolescence puis rendue à sa famille du ghetto de Bayonne, est la descendante des suppliciées. Elle

\footnotetext{
${ }^{11}$ La conception de Lasry rejoint celle exposée par Shmuel Trigano dans son article «Le bouc émissaire. En finir avec 1'expiation'», Pardès, 2002/1, n ${ }^{\circ}$ 32-33. https://www.cairn.info/revue-pardes-2002-1-page-117.htm.

${ }^{12}$ Lasry utilise l'orthographe espagnole originelle du patronyme de l'héroïne que nous désignerons ultérieurement comme Esther.
} 
doit fuir pour avoir enfreint l'interdiction de commerce privé frappant les Juifs. Déguisée en homme et sous un nom d'emprunt, elle tente vainement, pendant cinq ans, d'atteindre Amsterdam, Jérusalem du nord, errant sur la côte atlantique française entre Biarritz et Saint-Malo. En 1738, elle est finalement embarquée de force pour la Nouvelle-France à La Rochelle par les sbires de la Compagnie des Indes, à l'époque où seuls les catholiques étaient admis. Démasquée comme juive et femme à son arrivée à Québec, refusant d'être baptisée, elle est rapatriée en France un an plus tard aux frais du roi Louis XV.

Dans le roman, les Noirs et les femmes sont eux aussi représentés comme des victimes. Esther frémit d'horreur à la vue des malheureux esclaves dans la cale du navire, enchaînés et nourris de force. En Bretagne, elle assiste, sous le déguisement masculin qui la protège, à l'attaque d'une femme accusée de sorcellerie par la foule déchaînée. Condamnée sous prétexte qu'elle a jeté le mauvais œil sur une enfant épileptique, la malheureuse est sauvée du bûcher in extremis par un prêtre. Lors de la messe de minuit à laquelle Esther est obligée d'assister, à Saint-Malo, la crèche reconstituant la naissance de Jésus suscite en elle des pensées mêlées. À ce stade, comme c'est parfois le cas dans ses romans, l'auteur lui-même, témoin et commentateur, intervient dans un aparté inscrit en italiques, pour évoquer les persécutions menées contre les Juifs au nom de la foi chrétienne au cours de l'Histoire :

Qu'on se souvienne de Kielce en Pologne, pas au Moyen Âge, pas au dixhuitième siècle d'Esther Brandeau, non, par un beau dimanche ensoleillé, un 4 juillet 1946, un an après les horreurs de la Deuxième Guerre mondiale et le cauchemar d'Auschwitz! (JNF 240)

Le rappel du massacre de quarante-deux Juifs, survivants de la Shoah, sous prétexte de l'absence temporaire de trois enfants chrétiens, situe dans la dimension historique la prise du Juif comme bouc émissaire responsable de tous les maux. La suite du commentaire de l'auteur explique la position de l'héroïne, victime de la culture du silence instaurée par l'Église. Prenant le parti d'Esther, Lasry dénonce les « papes et princes », qui « connaissaient parfaitement la vérité » mais « ne faisaient rien », parce qu' « il est plus facile d'accuser la collectivité des Juifs pour la mort d'un seul de leurs frères » (JNF 241). L'intervention de l'auteur constatant que le Juif est toujours pris comme bouc émissaire, même après la Shoah, a pour effet de situer dans le présent les sévices subis dans le passé. 


\section{L'enquête sur le bouc émissaire}

Les deux autres romans de Lasry, qui relèvent du genre policier, se déroulent à notre époque. Les protagonistes sont des écrivains juifs accusés de meurtre et mués en détectives pour prouver leur innocence, retrouver le véritable criminel et confondre leurs détracteurs. Dans Don Juan et les moulins à vents, Henry-Aharon $\mathrm{Wise}^{13}$, journaliste montréalais et juif traditionaliste marié à Ruth, québécoise non juive, est amoureux de Naomi, belle et jeune convertie au passé douteux, mère de quatre enfants de pères différents. Lorsqu'il la trouve morte étranglée sur le sol de sa cuisine, il s'effondre sous l'effet d'une commotion cérébrale. Soupçonné du meurtre mais innocenté, il finit par retrouver le meurtrier. Naomi, socialement marginale, correspond au profil typique de la femme victimisée. Elle est doublée par un autre personnage féminin apparaissant brièvement dont Henry, psychologue improvisé, perçoit la détresse affective. Lorsque la même réapparaît à la fin, Ruth fait le lien avec Naomi qu'elle admire pour son courage. Par un effet d'emboîtement, Ruth, professeure d'ethnologie accaparée par les problèmes du Tiers Monde, et Naomi, pauvre, charitable et vivant d'expédients, ont en partage leur souci humanitaire. Naomi s'occupe de personnes encore plus défavorisées, la jeune paumée, "petit chaperon rouge » exploitée par un " grand méchant loup-proxénète » (DJ 85), les gens âgés et les mortes auxquelles elle rend bénévolement les derniers devoirs ${ }^{14}$. Autre exemple d'emboîtement de figures de défavorisés, les immigrants originaires d'Asie du Sud-est et d'Amérique du Sud, bloqués chez eux par la tempête, ostracisent Naomi par leurs regards soupçonneux. À l'opposé de la charité et de la compassion caractérisant Naomi, son père adoptif, Tim Sutter, suisse d'origine, a assisté en témoin passif, pendant la Seconde Guerre mondiale, au refoulement suivi de fusillade d'enfants juifs tentant de se réfugier en Suisse. En étranglant Naomi convertie au judaïsme, il actualise sa complicité avec les criminels nazis.

Lasry fait remonter l'action aux origines de l'humanité. Le roman s'ouvre sur un condensé de la création du monde au premier chapitre de la Genèse : le paradis avant la faute, le péché d'Adam sous l'impulsion

\footnotetext{
${ }^{13}$ Nous désignerons ultérieurement le personnage comme Henry.

${ }^{14}$ Ruth et Naomi sont toutes deux des Don Quichotte au féminin. L'allusion au livre biblique de Ruth est claire. Voir à ce sujet Simone Grossman, «Transculture et judéité dans Don Juan et les moulins à vent de Pierre Lasry », Canadian Jewish Studies / Études Juives Canadiennes, Volume XXV, 2017, p. 79-92.
} 
d'Ève la tentatrice, l'apparition du mal et le premier meurtre. Une vision paradisiaque est décrite, la journée merveilleuse, à peine automnale, au début du mois de Tichri ${ }^{15}$ où le monde a été créé. Dans le même ordre que dans le modèle biblique, un souffle d'air précède le soleil, le ciel bleu, le lac, le feuillage des arbres, puis les animaux de l'air et de l'eau, et enfin le couple de sexagénaires formé par Ruth, Québécoise, et Henry, Juif venu d'ailleurs, en posture tendre sur un banc au bord de l'eau. La ressemblance à la source s'arrête là, puisque l'idylle est dissipée par l'apparition de la tentatrice jeune et blonde, métaphore du mal. La duplicité d'Henry se mesure au regard oblique et convoiteux, fauteur de chute morale. Le paradis, âge d'or de l'innocence, est en passe de se muer en enfer. Comme dans un film, la jeune blonde disparaît du champ de vision remplacée en fondu enchaîné par "la femme qui va suivre » (DJ 15), s'agissant de la belle Naomi.

Le motif de l'accusation contre Henry tient à la position équivoque dans laquelle la police le trouve, évanoui, son corps recouvrant le cadavre de Naomi. Sa chute émanait de son désir de regarder la morte de trop près. La scène primaire du récit fait surgir la solution de l'énigme dans la vision-éclair où l'action défile comme un film. Voulant appliquer la théorie de la persistance rétinienne, selon laquelle l'agresseur est visible dans les pupilles de sa victime, il regarde dans les yeux de la morte et voit clairement le visage du meurtrier avant de subir lui-même l'agression qui lui fait perdre l'équilibre.

Le visage de l'étranger s'agrandit et les iris noirs de Naomi envahissent l'œil d'Henry comme une marée de goudron qui déferle. Il tombe en avant contre elle et se confond à la nuit. (DJ 25) ${ }^{16}$

Henry n'aura de cesse de retrouver le meurtrier dont il a entr'aperçu le visage. Le récit se déroule comme une vision fantasmatique. Il éprouve «l'étrange sentiment d'évoluer au ralenti, détaché de ce monde, derrière la vitre blindée de l'aquarium d'un mauvais rêve » (DJ 22). Sa chute l'entraîne dans l'aquarium, dispositif ${ }^{17}$

\footnotetext{
${ }^{15}$ Premier mois du calendrier hébraïque, correspondant à septembre-octobre.

${ }^{16}$ Le « goudron » sortant des yeux de Naomi est pour Henry l'encre de son écriture.

${ }^{17}$ Jean-Louis Baudry, « Le dispositif : approches métapsychologiques de l'impression de réalité », Communications, $\mathrm{n}^{\circ} 23,1975, \mathrm{p}$. 7. Le dispositif, dans une terminologie platonicienne et freudienne, est aussi un médium filmique, un appareil de «la projection et dans lequel le sujet à qui s'adresse la projection est incluse » [8][8], Ibid., p. 58. https://books.openedition.org/pupo/3494?lang=fr
} 
optique anticipant sur l'eau profonde d'où, plus tard, " comme un plongeur de cinéma » (DJ 288), il vise et tue l'assassin de Naomi.

Dans l'intervalle qui s'écoule entre le crime et la résolution de l'énigme, Henry, d'abord suspecté, est devenu une victime. Paradoxalement, en tant que journaliste, il est aussi enquêteur. Il a joué au détective pour élucider le mystère de la vie nocturne de Naomi, découvrant alors ses activités charitables. Lorsqu'elle gît, morte, Henry est pris à son propre jeu de voyeur. Blessé dans sa chute, il est humilié et malmené par les policiers qui l'emmènent à l'hôpital en le brutalisant et en le traitant de « vieux sale » (DJ 31), accusation qui l'atteint au profond de lui-même.

\begin{abstract}
Même si on me déclare innocent, comment vais-je me divertir de ce vêtement de honte qui colle à ma peau comme un vieux pansement sur une plaie purulente ? [...] La honte n'est pas quelque chose qu'on peut envoyer au dégraissage, sans y laisser un peu de sa peau et de sa chair, et une partie non-négligeable de son âme. (DJ 32)
\end{abstract}

Soucieux de sa réputation de journaliste et de la bonne opinion de son épouse, il s'évanouit et sombre, inconscient, dans une absence temporaire qui le libère de ses soucis.

\title{
L'écrivain, bouc émissaire pornographe
}

L'homme qui n'avait rien à dire se déroule en Floride et au Québec. L'écrivain juif Alex Portugais ${ }^{18}$, accusé de l'assassinat de Jacinthe Gélinas, jeune Québécoise d'origine juive, ne se souvient de rien. Bien que la morsure au cou de la victime ne puisse lui être imputée, vu la nonconformité de sa dentition avec les marques de l'agression, il est néanmoins condamné à douze ans de prison. Libéré plus tôt pour bonne conduite, il épouse la fille d'un riche marchand d'armes décidée à l'aider à prouver son innocence. Elle lui prête main-forte pour récupérer les microfilms des caméras de surveillance attestant son innocence. Au terme de procès moralement très éprouvants, il est exonéré de l'accusation.

Comme pour Henry, la victimisation est déclenchée par la visualité. Prototype du Juif errant, parcourant le monde muni seulement de son tallith, châle de prière, de ses phylactères et de son livre de prière, Alex

\footnotetext{
${ }^{18}$ Nous le désignerons comme Alex.
} 
est un vagabond sans feu ni lieu, à la mauvaise vue et ignorant de son identité. Le premier chapitre, très bref, incluant des indications spatiotemporelles, «Au Café Vert, Bal Harbour, décembre 2007 », circonscrit l'essence identitaire de son investigation: «J'ai fouillé dans mon sac de voyage pour voir qui j'étais » (HD 7). Vu l'inefficacité des gouttes pour les yeux, l'écriture prend le relais de la vision bien qu'il soit réduit à écrire son «mot pour [se] réinventer» (Ibid.) sur le verso du menu. Son "gribouillage » illisible, contrastant avec le «contenu littéraire » du menu au recto (HD 8), figure l'énigme voilée. Obsédé par le soupçon, qui pèse sur lui, du meurtre d'une jeune femme dans un motel en Floride, il est renvoyé par sa mémoire embrouillée à un autre motel aux « photos en couleurs de piscine sous les cocotiers et de mer bleue » et à l'enseigne du «Café Vert Cacher » (HD 10) où il a séjourné avec son épouse. Sur les photos, les lieux et les époques sont confondus. Autre instrument optique de brouillage, le "miroir embué » de la salle de bains (Ibid.) voile, au lieu de le refléter, le plateau-café blanc et les sachets roses de faux sucre qui lui rappellent la prison et le ramènent mentalement au meurtre qu'il n'a pas commis. Il évolue dans un flou mental sans comprendre pourquoi on le prend pour un «meurtrier pornographe ${ }^{19}$. Il argue qu'il n'est « ni meurtrier ni pornographe » (HD 75) bien qu'il apparaisse comme la « vedette principale » d'un « film porno » (HD 77). Plus tard, au terme de son investigation, il découvre les microfilms dissimulés contenant les images du scénario monté de toutes pièces par un producteur de films pornographiques, beau-frère de la victime (HD 95). Le réel et le fantasmatique se mêlent dans le rêve où il se voit endormi. Son visage couvert d'un masque pointe sur le sommeil artificiel où l'a plongé la drogue administrée à son insu. L'action se fait visuelle, la première image effacée en fondu enchaîné par la seconde. Le dédoublement de l'homme masqué en deux instances narratives interchangeables fait de lui simultanément une victime et un voyeur.

L'histoire d'Alex se déroule sur le fond de l'antisémitisme. Retrouvant les microfilms qui prouvent son innocence, il dévoile sa relation avec les Gélinas, riche famille québécoise descendant de nouveaux-chrétiens, ses lointains coreligionnaires :

Il y a des Hébert, des Lévesque, des Parizeau, des Bourgault et des Martineau d'origine juive dans ma famille, et ça ne les empêchait pas de haïr les Juifs. Par centaines, ceux qui ont fui l'inquisition se sont réfugiés en Nouvelle-France

\footnotetext{
${ }^{19}$ Le sous-titre du roman est « Portrait de l'auteur en meurtrier pornographe ».
} 
comme Nouveaux-Chrétiens vers 1650. C'est facile à vérifier aujourd'hui. Un petit test d'ADN... (HD 85) ${ }^{20}$

Drapé dans son " tallith rapiécé », il procède à un retour mental sur le même "vêtement à franges » offensant ses codétenus, les «Black Muslims » (HD 49). Suit un développement sur leur " révision militante de l'histoire » d'après laquelle « les Juifs s'étaient tous rétroactivement convertis à l'Islam à cause de leur ancêtre Ibrahim, Abraham » et de ses descendants, «tous devenus musulmans deux mille ans avant la naissance l'Islam », y compris « un autre Juif, nommé Yéchu, divinisé par certains Chrétiens, qui, lui aussi s'était converti à l'Islam, sans le savoir »(Ibid.). En prison, le 11 septembre 2001, Alex maltraité rit de son propre sort. Un débat théologique s'ensuit entre l'aumônier, Rav Zusche, les Muslims et les Québécois de souche, catholiques. Confronté à la mise en accusation des Juifs tenus responsables des attentats du 11 septembre, Alex affirme pour sa défense et la leur, qu' :

[...] on ne peut pas être libre si l'on voit les Juifs comme responsables des malheurs du monde. On vous dit qu'ils sont derrière toutes les guerres? Ils en sont les premières victimes, avait dit Rav Zusche. Ils ont assassiné Dieu? Ils l'ont plutôt révélé au monde ! (HD 52)

Le débat est conclu par les sourates confirmant le statut du peuple juif, «l'élu d'Allah » (HD 54), préludant à la future réhabilitation d'Alex.

\section{Le bouc émissaire et la culture du silence}

Sous forme d'allusions et de commentaires, l'actualité internationale sert de fond à l'action de Don Juan et les moulins à vent. Henry subit le contrecoup de la désinformation rendant les Juifs coupables du terrorisme, de l'attentat contre les tours de Manhattan, des actions de l'armée israélienne et de la réalité sociale sordide. Il est renvoyé du journal à cause de ses prises de position pro-israéliennes. Les véritables tenants et aboutissants du meurtre de Naomi doivent être tenus secrets et la bonne conscience de ses détracteurs sauvegardée. Si Henry, cible idéale de l'accusation en tant que Juif non conformiste, retrouve l'assassin, ce n'est pas grâce à la police, représentant la société. À la fin

\footnotetext{
${ }^{20}$ Le personnage renchérit en affirmant que le nom québécois « Gélinas » est « une contraction du sobriquet Juif Élie » (HD, p. 85).
} 
de son enquête, simulant un accident, il se mue en justicier et décapite Sutter l'assassin.

Sutter faisait partie de cette bande de salauds qui envoient chaque année des centaines d'enfants dans les hôpitaux psychiatriques, alors que c'est eux, les salauds, qu'on aurait dû enfermer les premiers. (DJ 296)

Henry, mystificateur doublé d'un justicier, feint l'ignorance. Pardelà le meurtre de Naomi, victime innocente, il venge les enfants juifs assassinés. À rebours de l'ambulance hurlante où est «emprisonné » Henry accusé à tort du péché commis par un autre, celle emportant les restes de l'assassin complice des nazis quitte discrètement les lieux. La vengeance revêt la valeur d'un acte moral accompli par Henry redresseur de torts.

À la fin de L'homme qui n'avait rien à dire, la demande en recours d'Alex aboutit à son acquittement. Par défaut, l'image montre qu'il n'était pas seul dans la chambre lors du crime, mais en compagnie de deux cinéastes. Le fait est corroboré par les microfilms où on le voit endormi sur un sofa, " preuve graphique » (HD 190) de son innocence. $\mathrm{Au}$ terme de procédures minutieuses, le procès se clôt sur le non-lieu.

Un non-lieu. [...] Tout ce qui m'est arrivé n'est pas arrivé. [...] tout ce que les chroniqueurs imbéciles de chaque époque de ma nouvelle histoire ancienne ont clamé haut et fort comme des vérités, tout ça, des fantasmes, des projections sur écran déjà pollué $[\ldots]$ par la peur de disparaitre que chacun de nous essaie d'évacuer en la projetant hors de soi, sur les autres, les étrangers, moi, vous. (HD 198-199)

La culpabilité dont il a été officiellement dégagé lui colle encore à l'âme, devant être évacuée par d'autres moyens qu'un procès qui l'innocente. L'accusation dont il a été exonéré ne se limite pas à sa personne privée mais vise l'ensemble de la collectivité juive dans l'Histoire.

\section{La parole réparatrice du « bouquin émissaire » - bouteille à la mer}

Dans son article «Réflexion sur les marranes », Lasry définit le marrane, par-delà son sens historique, comme chaque Juif vivant dans l'exil « de la parole et de la conscience ». Le «midbar», signifiant « désert » en hébreu, est le « lieu du silence », désert de la parole d'où le 
Juif-marrane, à l'égal de l'artiste, est appelé à « réparer le monde $»^{21}$. Pour Alex, la réparation est l'écriture libérée qui le fera renaître innocent.

Il faut libérer l'écriture [...] se lâcher dans le vide d'un autre moi [...] cette défroque de culpabilité que j'ai si longtemps habitée s'est presque détachée de mon corps. (HD 199-200)

Investi de la «mission» de «réparer» (DJ 302), attendant le moment adéquat pour tuer Sutter, Henry plongé dans l'eau comme dans un bain rituel improvisé ${ }^{22}$, se purifie symboliquement de l'accusation portée contre lui. Liés intrinsèquement à la métaphore aquatique salvatrice, la réparation de la faute et le renouvellement salvateur sont la finalité de l'écriture.

Et puisque j'utilise des mots comme instruments de ma survie, mots radeaux pour ici-bas [...] pourquoi ne pas commencer par dire que chaque mot est une occasion de changer [...] chaque parole un arbre de vie dont nous devrons nousmêmes manger les fruits. (DJ 12)

La Thora, «arbre de vie ${ }^{23}$ est prolongée dans la parole de l'écrivain juif, jaillissant parfois comme un «cri [...] distillé de la toile d'un peintre, des planches d'un théâtre, d'un décor de cinéma, de la feuille blanche d'un écrivain » (HD 157).

Réitérant le geste du grand-prêtre précipitant le bouc émissaire dans l'abîme du désert, en expiation des fautes de la collectivité, l'écrivain et artiste juif lance son «bouquin émissaire » (HD 166) ou « bateau-livre » (HD 169) dans la mer, dotant sa parole du pouvoir de rédimer un monde imparfait. Le livre refait le parcours du Juif persécuté vers la rive opposée de l'océan, «à l'autre bout d'un monde sauvage, en Amérique, là où une Europe meurtrière va renaître d'un sang nouveau », où il va "guérir» ses blessures (DJ 9-10). L'art, qu'il soit un écrit, un tableau ou un film, répare les fautes imputables à un monde imparfait. Le but véritable de l'écriture n'est pas de «dire quelque chose » (HD 7) mais de « remédier à la condition faite à tous ceux qui comme le Juif, l'étranger, l'esclave, le Noir, concentrent sur eux le regard mauvais et la

\footnotetext{
${ }^{21}$ Pierre Lasry, « Réflexion sur les marranes », dans Jean-Claude Lasry, Joseph Lévy et Yolande Cohen (dir.), Identités sépharades et modernité, Québec, Les Presses de l’Université Laval, 2007, p. 73-80. La citation est à la p. 75.

${ }^{22}$ Allusion au mikveh, bain rituel au pouvoir purificateur reproduisant celui du déluge d'où le monde ressort neuf.

${ }^{23}$ Proverbes, 3, 18 http://www.mechon-mamre.org/f/ft/ft2803.htm
} 
haine des autres ${ }^{24}$. Alex interpelle « l'éditorialiste » qui, pour « séduire ses clients, ses lecteurs, et surtout son patron» (HD166), soutient le boycott des Juifs, que ce soient les musiciens trop doués de l'orchestre symphonique de Montréal ou les trop nombreux titulaires du prix Nobel. Le même éditorialiste attaque les Hassidim d'Outremont, boucs émissaires d'une presse désireuse d'attiser l'opinion publique contre des victimes toutes désignées ${ }^{25}$. Or la fonction de l'écrivain juif consiste tout d'abord à s'auto-critiquer pour ne pas entrer dans la logique du bouc émissaire. «Ah, mais pourquoi m'en prends-je à ce pauvre chroniqueur qui joue au jeu du bouc émissaire. Pourquoi en fais-je mon propre bouc émissaire ?» (HD 166), s'interroge Alex s'interdisant d'imputer trop facilement la responsabilité du mal à autrui, et appliqué à démêler l'enchaînement des causes et des effets aboutissant à la désignation de boucs émissaires.

En guise de conclusion, reportons-nous à la première de couverture de L'homme qui n'avait rien à dire où un remake du Cri d'Edvard Münch est enchâssé entre la phrase-titre ekphrasis et le nom de l'auteur ${ }^{26}$. Seules apparaissent la tête et les mains du personnage, peintes dans le même bleu-vert que le fjord dans le tableau de Münch. Sur la page de garde, le sous-titre, Portrait de l'auteur en meurtrier pornographique, établit le rapport entre le meurtre, le récit et l'image. Suivant immédiatement, l'incipit, « Je suis écrivain. Je n'ai rien à dire » (HD 7), répète la phrasetitre accompagnée par l'image du personnage qui recouvre ses oreilles de ses mains. Le jeu de reflets entre la phrase-titre du roman et l'allusion picturale ${ }^{27}$ déjoue le silence. Un autre langage est créé par l'inversion des couleurs, le bleu aquatique du visage originellement blanc chez Münch. Contrairement à ce dernier, " phagocyté » par le « décor poisseux » où il a perdu ses repères, hurlant $l^{\prime} \ll$ inaudible cri qui nous assaille ${ }^{28}$, l'homme du remake de Lasry, où le bleu efface la blancheur cadavérique,

\footnotetext{
${ }^{24}$ P. Lasry, « Réflexion sur les Marranes », op. cit., p. 79.

${ }^{25}$ Pour prendre connaissance des controverses pour lesquelles les Juifs hassidiques d'Outremont ont été pris à partie, consulter les sites internet suivants : http://outremonthassid.com/?cat=36\&lang=f r_fr

https://ici.radio-canada.ca/nouvelle/1001057/hassidim-referendum-outremontinterdiction-lieux-culte

${ }^{26}$ Lasry, également peintre, est l'auteur du remake.

27 Voir à ce sujet Liliane Louvel, Le tiers pictural, Pour une critique intermédiale, Rennes, Presses Universitaires de Rennes, 2010.

${ }^{28}$ Pierre Fresnault-Deruelle, «Cet inaudible cri qui nous assaille », Musée critique de la Sorbonne. http://mucri.univ-paris1.fr/cet-inaudible-cri-qui-nous-assaille/
} 
est doté de langage. Une relation nouvelle, intronisée par l'inversion comme sur un négatif photographique, transforme l'imparfait de la phrase-titre en présent de l'écriture. Grâce à son récit rétablissant la vérité et la justice, Alex, "criard muet» (HD 157), a cessé d'être une victime bâillonnée au visage blafard. Doté de voix, il est portraituré criant son innocence, son visage osseux et desséché revitalisé par la mouvance bleu-vert de l'eau. Le dynamisme dialogique de l'image remédie au silence tandis que, de l'application du bleu marin au personnage désespéré de Münch, se dégage une image renouvelée du Juif, artiste et marrane.

$* * * * * * * * * *$

\section{Euvres citées}

- Baudry Jean-Louis, « Le dispositif : approches métapsychologiques de l'impression de réalité », Communications, n 23, 1975, p. 56-72.

- Fresnault-Deruelle Pierre, "Cet inaudible cri qui nous assaille ». Musée critique de la Sorbonne. http://mucri.univ-paris1.fr/cet-inaudible-criqui-nous-assaillel

- Grossman Simone, "Transculture et judéité dans Don Juan et les moulins à vent de Pierre Lasry », Canadian Jewish Studies / Études Juives Canadiennes, Volume XXV, 2017, p. 79-92.

- Lasry Pierre, «Les Québécois sont tous un peu marranes », La Presse, Montréal, 24 juin 2001.

http://collections.banq.qc.ca:81/lapresse/src/cahiers/2001/06/24/B/82812 20010624B.pdf

- Lasry Pierre, Une Juive en Nouvelle-France, Montréal, Éditions Midbar, 2004 [2000].

- Lasry Pierre, "Réflexion sur les marranes », dans Jean-Claude Lasry, Joseph Lévy et Yolande Cohen (dir.), Identités sépharades et modernité, Québec, Les Presses de 1'Université Laval, 2007, p. 73-80.

- Lasry Pierre, Don Juan et les moulins à vents, Montréal, Éditions du Marais, 2008.

- Lasry Pierre, « Scapegoat», J Mag, Montréal, novembre 2008. Sans pagination. Document fourni par l'auteur. 
- Lasry Pierre, L’homme qui n'avait rien à dire, Montréal, Éditions Midbar, 2011.

- Trigano,Shmuel, "Le bouc émissaire. En finir avec 1'“expiation'», Pardès, 2002/1, n 32-33, p. 117-127.

\section{Filmographie}

Shylock. Réalisateur: Pierre Lasry, National Film Board of Canada, 1999. 